is naturaleza 
Para la publicación de este número se ha contado con la ayuda

financiera de las siguientes instituciones:

Departamento de Filosofía y Lógica y Filosofía de la Ciencia de la Universidad de Sevilla

Facultad de Filosofía de la Universidad de Sevilla 


\section{NATURALEZA Y LIBERTAD}

Revista de estudios interdisciplinares

Número 4

Málaga, 2014

Esta revista es accesible on-line en el siguiente portal:

http://grupo.us.es/naturalezayl 


\section{Naturaleza y Libertad}

Revista de estudios interdisciplinares

Número 4

ISSN: 2254-96682014

Directores: Juan Arana, Universidad de Sevilla; Juan José Padial, Universidad de Málaga; Francisco Rodríguez Valls, Universidad de Sevilla.

Secretaria: Avelina Cecilia Lafuente, Universidad de Sevilla.

Consejo de Redacción: Jesús Fernández Muñoz, Universidad de Sevilla; José Luis González Quirós, Universidad Juan Carlos I, Madrid; Francisco Soler, Universität Dortmund / Universidad de Sevilla; Pedro Jesús Teruel, Universidad de Valencia; Héctor Velázquez, Universidad Panamericana, México.

Adjunto a la redacción: Miguel Palomo, Universidad de Sevilla

Consejo Editorial: Mariano Álvarez, Real Academia de Ciencia Morales y Políticas; Allan Franklin, University of Colorado; Michael Heller, Universidad Pontificia de Cracovia; Manfred Stöcker, Universität Bremen; William Stoeger, University of Arizona.

Consejo Asesor: Rafael Andrés Alemañ Berenguer. Universidad de Alicante; Juan Ramón Álvarez, Universidad de León; Luciano Espinosa, Universidad de Salamanca; Miguel Espinoza, Université de Strasbourg; Juan A. García González, Universidad de Málaga; José Manuel Giménez Amaya, Universidad de Navarra; Karim Gherab Martín, Urbana University, Illinois; Martín López Corredoira, Instituto de Astrofisica de Canarias; Alfredo Marcos, Universidad de Valladolid; Marta Mendonça, Universidade Nova de Lisboa; Javier Monserrat, Universidad Autónoma de Madrid; Leopoldo Prieto, Colegio Mayor San Pablo, Madrid; Ana Rioja, Universidad Complutense, Madrid. Madrid; José Luis González Recio, Universidad Complutense, Madrid; Javier Serrano, TEC Monterrey (México); Hugo Viciana, Université Paris I; Claudia Vanney, Universidad Austral, Buenos Aires; José Domingo Vilaplana, Huelva.

\section{Redacción y Secretaria:}

Naturaleza y Libertad. Revista de estudios interdisciplinares. Departamento de Filosofia y Lógica. Calle Camilo José Cela s.n. E-41018 Sevilla.

Depósito Legal: MA2112-2012

욜 954.55.77.57 Fax: 954.55.16.78. E-mail: jarana@us.es

(C) Naturaleza y Libertad. Revista de Filosofía, 2014 


\section{ÍNDICE}

\section{ESTUDIOS}

Manuel Alfonseca (Universidad Autónoma de Madrid), Diseño inteligente, evolución al azar, o evolución providencial....... ..11

Paul Gilbert, S J (U. Gregoriana, Roma), Deseo de conocer ..............................................27

Javier Hernández-Pacheco (U. de Sevilla), Evolución, erotismo y origen de las especies. De vuelta desde Darwin a Platón y Aristóteles.

Miguel Ángel Herrero (U. Politécnica de Madrid), La formación de los conceptos científicos. De Grosseteste a Galileo.

Ma Teresa Pelacho López (Zaragoza), Otra autonomía de la ciencia. Reflexiones a la luz del pensamiento de Serguei Kara-murzá.......

Juan Fernando Sellés (U. de Navarra), Estudio de las tesis centrales de Millán-Puelles sobre la libertad....... 189

Francisco Soler Gil (U. de Sevilla), El naturalismo y la tentación de las extrapolaciones omnicomprensivas.

Héctor Velázquez Fernández (U. Panamericana, México), En torno a la naturalización de la conciencia sugerida por Daniel Dennett

\section{SECCIÓN BIBLIOGRÁFICA}

Maria Camila Gallego: Falsos saberes.

Juan Arana: Bernardino Telesio, La naturaleza según sus propios principios. 


\title{
DESEO DE CONOCER
}

\section{Paul Gilbert, S J \\ Pontificia Universidad Gregoriana, Roma}

\begin{abstract}
Resumen: ¿Es el conocimiento una actividad de carácter únicamente potente e invasivo? Una mirada sobre la experiencia del conocimiento invita, por el contrario, a reconocer un momento pasivo - $\mathrm{O}$ una disposición a acoger aquello que es- para entenderlo mejor. Esta atención implica una predisposición afectiva. El artículo recuerda que esta "pasividad" ha sido reconocida a lo largo de toda la historia de la filosofía. Conviene, no obstante, precisar el vocabulario y distinguir entre afecto, emoción, pasión y sentimiento.
\end{abstract}

Palabras clave: conocimiento, afecto, pasión, emoción, sentimiento, pasividad, lenguaje

Abstract: Is knowledge an activity of a powerful and invasive nature? An insight in to the experience of knowledge invites, on the contrary, to recognize a passive moment - or a disposition to accept what it is - for a better understanding. This attention involves an affective predisposition. The article reminds that this "passivity" has been recognized throughout the whole history of philosophy. It is advisable, however, to specify the vocabulary and distinguish between motion, passion and feeling.

Keywords: Knowledge, affection, passion, emotion, feeling, passivity, language

Recibido: 12/06/2012. Aprobado: 15/03/2013

El conocimiento efectivo no está exento de cierto deseo; de hecho, aquél podría constituir sólo una modalidad de ese deseo, a la vez intelectual y afectivo, que consideraremos el aspecto más fundamental y determinante de nuestro ser humano. Aristóteles escribe en la primera línea de su Metafi- 
Naturaleza y Libertad. Revista de estudios interdisciplinares. Número 4, 2014. ISSN: 2254-9668

sica ${ }^{1}$ que «todos los hombres desean por naturaleza ver con claridad». Un deseo natural precede al conocimiento, y le impone su marca. Parece, sin embargo, evidente que el conocimiento efectivo de la realidad deba poner entre paréntesis todo deseo, sobre todo porque da la impresión de que éste siempre ha vivido bajo la presión de algún afecto, o, más precisamente, de una conciencia secreta de carencia de algo; y no existiría conocimiento verdadero alguno si no es completo y definitivo. Pero el conocimiento emprende siempre su investigación, comienza a ponerse en acto, cuando la conciencia se sorprende ante un evento que no consigue asumir en su representación sistemática del mundo. Ahora bien, ¿existiría un deseo carente de afecto o sentimiento alguno? Y aunque en verdad los afectos no son buenos consejeros del conocimiento, ¿podría acaso el conocimiento prescindir de ellos? Uno de los cometidos del trabajo que propongo a continuación será, por tanto, precisar y distinguir diferentes aspectos del afecto que forman parte del deseo de conocer, principalmente: el afecto y el sentimiento. Tras la lectura del artículo reciente de un pensador italiano sobre la estructura fundamental del deseo (2), atravesaré velozmente algunas etapas de la reflexión filosófica al respecto (3); terminaré con una propuesta sistemática sobre afecto y sentimiento (4); iniciaré, no obstante, mi discurso considerando brevemente la etimología de dos términos principales: "deseo" y "afecto"(1).

1 Aristóteles, Metafísica, A/I, 1 (980a20): «Pantes antbropoi tou eidenai oregontai fusei». 
Naturaleza y Libertad. Revista de estudios interdisciplinares. Número 4, 2014. ISSN: 2254-9668

\section{ETIMOLOGÍA DE LAS VOCES “DESEO” Y “AFECTO”}

Comienzo mi exposición con una referencia a la etimología de los términos "deseo" y "afecto", dado que cada uno de ellos posee una larga historia que, aunque se nos esconde, pervive todavía hoy, aportando significado y orientación a los dinamismos de nuestras culturas latinas.

El término "deseo" procede, en parte, del latín "sidus", voz que significa un conjunto de estrellas que, vinculadas las unas con las otras, presentan metafóricamente ciertas "figuras", por ejemplo, la «osa mayor», «el can mayor», etc., mientras que la voz en singular "estrella" indica un cuerpo celeste brillante y aislado. El "de" de "deseo" es privativo. El término "deseo" revela, por tanto, la privación de una visión de la forma, es decir, de un significado sobre el cual la mente pueda apoyar su investigación. El "deseo", que procede, por tanto, de la toma de conciencia de la carencia de un sentido, se opone a la "consideración", que significa el acto de unir "estrellas" de modo que resplandezcan juntas en un significado o una forma, esto es, la "morphe" de los Griegos. Notemos que el término "deseo", cuyo significado indica privación, connota también la actividad de la investigación que pretende colmar tal privación. Y si la privación es la "dunamis" aristotélica, el "deseo" ofrece propiamente un nombre para la "energeia" del Estagirita.

Por su parte, el sustantivo "afecto" procede de dos fuentes. Una primera podría ser el verbo latino "afficere" ("tocar", "conmover" [por ejemplo el espíritu], "influir sobre”, “disponer a”, y al fin "adornar de”). Distingamos en este 
Naturaleza y Libertad. Revista de estudios interdisciplinares. Número 4, 2014. ISSN: 2254-9668

verbo la preposición "ad" ("hacia”) y el verbo "facere" ("hacer", "operar", "actuar", "producir"). Por extensión, afficere significa «imponer su marca sobre otro», y, en consecuencia, «hacer enfermar», o, más sutilmente, «hacer sufrir a otro una acción mía». Una segunda fuente, muy similar a la anterior, sería "affectare", es decir, «buscar con ansia a fin de alcanzar alguna cosa», y así, «ponerse a trabajar». Toma a continuación un significado de más peso: «abusar de ornamentos», «utilizar artificios de exquisitez en las palabras y en los modos, mediante los cuales uno se esfuerza para hacerse atribuir ciertas cualidades de las que carece». El participio pasado pasivo, "affectus", que podría venir de afficere o de affectare, significa «que ha sufrido un golpe», «que ha sido tocado». Este "pasivo" provoca sin embargo una reacción consciente por parte del tocado. Con todo, se podría ya esbozar una posible definición: el afecto es una «manera de sentir», una «pasión del alma excitada por un movimiento interno», una «inclinación a amar o a odiar, a la compasión, a la ira, etc.» ${ }^{2}$. A este significado le sigue otro de carácter económico: «affectare un bien» significa «destinarlo a un determinado destinatario»o, en una empresa o una administración, «destinar alguno a un trabajo concreto».

2 Diccionario etimológico on line. 
Naturaleza y Libertad. Revista de estudios interdisciplinares. Número 4, 2014. ISSN: 2254-9668

\section{UNA PROPUESTA RECIENTE SOBRE EL “AFECTO”}

Los temas del deseo y del afecto han estado presentes a lo largo de la historia de la reflexión. Antes de interesarme por ella, me detendré sobre un artículo reciente ${ }^{3}$ que tiene la virtud de ser sintético y de presentar al mismo tiempo los aspectos fundamentales de la problemática de los afectos.

Adriano Fabris, profesor de la Università degli studi di Pisa, adopta sobre el tema del afecto una perspectiva global precisa: los afectos son, por supuesto, formas de participación subjetiva, que tienen, sin embargo, un significado a priori, esto es, dado con precedencia, antes de que se produzca el acceso a la conciencia de estar afectado. Tal sentido no resulta auto-determinado por la subjetividad, es decir, fijado por el movimiento de una mente deseosa y orientada por sí misma. Observamos antes de todo que el término latino "affectio" traduce de hecho el griego "pathos", que podría significar «estado de ánimo». Según Fabris, este estado de ánimo, el afecto, no procede de un sujeto cerrado en sí mismo; antes bien, como el propio Heidegger había entendido correctamente, se da «una posibilidad clara de relación entre el sujeto y los fenómenos» ${ }^{4}$. El pathos se sitúa entonces entre el sujeto y el objeto constituyendo así una mediación que resuena subjetivamente y que no

3 A. Fabris, «Il coinvolgimento degli affetti», en Fr. Botturi-C. Vigna (edd.), Affetti e legami, Milano, Vita e pensiero, 2004, pp. 23-36.

4 A. Fabris, «Il coinvolgimento degli affetti», p. 24. 
puede, por ello, ser descrita sólo "científicamente", es decir, como un objeto de ciencia totalmente al descubierto ante los ojos de un analista sin intereses, neutro. Para comprender el pathos, es necesaria una investigación referida al origen, es decir, de carácter filosófico, o mejor aún, fenomenológico, que considere la intencionalidad personal que responde a la provocación original de lo que adviene.

Según Fabris, el pathos posee además una característica esencialmente trascendental por cuanto, siguiendo la reflexión que, sobre el misterio, lleva a cabo Gabriel Marcel, el pathos está «ya presupuesto en cada una de sus eventuales tematizaciones» ${ }^{5}$. No puedo tematizar el pathos sin padecer del pathos mismo. Esta trascendentalidad, dada a priori o antes de su conocimiento temático y sintético, no es "susceptible de ser representada" o puesta como tesis, ya que, dice Fabris, el pathos «existe, se da» ${ }^{6}$, me acontece. No se conforma sólo a un cierto significado impuesto por los procedimientos científicos, y mucho menos al significado que procede de una perspectiva "trascendental” que pretendería proponer una estructura básica, inmóvil, inerte y descriptible. El pathos se produce en cambio junto a una "emoción”, un evento que dinamiza el alma. «Así que, [...] más que de "estados de 
Naturaleza y Libertad. Revista de estudios interdisciplinares. Número 4, 2014. ISSN: 2254-9668

ánimo" sería apropiado hablar de "movimientos" del ánimo: precisamente, de emociones» ${ }^{7}$.

Entonces surge la pregunta: ¿qué es lo que específicamente mueve la emoción? Para Descartes, ésta no sería sino un movimiento mecánico, un movimiento interior del alma, concebido, sin embargo, de forma metafórica como el movimiento de cada res extensa ${ }^{8}$. El mecanismo cartesiano parece pesar sobre todos los trabajos científicos modernos y contemporáneos. Para las neurociencias contemporáneas, por ejemplo, las emociones están dotadas ante todo de eficiencia, de acuerdo con las reglas precisas de la causalidad científica; el esquema científico causa-efecto parece imponerse de hecho de un modo absoluto ${ }^{9}$ toda investigación científica. Ahora bien, este esquema causal se interpreta espontáneamente como si fuese posible unir por un lado actividad (causa) y por otro lado pasividad (efecto, que sería la emoción). Lévinas invita a considerar los eventos que preceden a los conceptos analíticos de la ciencia física, criticando de esta forma la distinción de la causa y del efecto, de la actividad y de la pasividad. Los eventos afectivos son a la vez activos y pasivos. La pasividad afectiva implica, de hecho, una actividad, si bien, diversa de la actividad de la causa. El esquema causa (activa) / efecto

7 Ibid.

8 Véase R. Descartes, Les passions de l'âme, art. 27.

9 Véase A. Damasio, Descartes' Error. Emotion, Reason and the Human Brain, New York, Avn Books, 1995, p. 248. 
(pasivo) no sirve del todo en el ámbito propio del deseo. Es preciso tener en cuenta un matiz todavía más esencial, señalado por Lévinas. De hecho, en la experiencia humana, se da una "pasividad más pasiva que cualquier pasividad ${ }^{10}$, es decir, más pasiva que la pasividad del efecto.

Padecer (pathos) no es sólo sufrir: «la dinámica de la afectividad no puede, en absoluto, reducirse a un simple sufrir» ${ }^{11}$. La experiencia enseña que la solicitación del otro comporta «en sí misma, un contra-golpe y una adaptación», «sobre todo, porque el mismo hacerse pasivo, la misma disponibilidad a ofrecerse, asume el carácter de un actuar para una pasión». El auto-afecto supone al mismo tiempo la capacidad de actuar sobre sí, hasta el punto de poder, de este modo, definir el pathos como «una entrevista consigo mismo y con otro», y, por ello, un conjunto de actividad y pasividad.

Tal conjunto no es dialéctico, una articulación de dos momentos contrarios que se sucederían en relaciones alternas de las que resultaría un tercer elemento mediador que anularía en sí a los mediados; sino que los dos polos están de hecho siempre presentes a la vez el uno en el otro, llamándose uno a otro. Se entiende, de este modo, el carácter reflexivo del sentir: la auto-afección parece tener un papel esencial, más aun, originario. «Sentir es sentir algo

10 Citado por A. Fabris, «Il coinvolgimento degli affetti», p. 25.

11 Las citas de este párrafo están tomadas de A. Fabris, «Il coinvolgimento degli affetti», p. 26. 
y sentir que se siente algo, esto es, darse cuenta del sentir, sentiendo» ${ }^{12}$. Por tanto, no es posible que la razón se contente con una simple objetivación del sentir; la conciencia del evento de sentir algo, a partir de la cual la ciencia objetiva toma conscientemente su impulso, es la misma conciencia que advierte el sentir subjetivamente algo. La conciencia de ser subjetivamente en acto de sentir adviene adhiriendo al sentir efectivamente algún objeto, en ese mismo momento. La auto-afección del sentir que siente sentir algo constituye, por ello, la puesta en acto de «una especie de "colapso" de actividad y pasividad, de "sujeto" y “objeto" en una inmediata auto-relación»"

Sentir el cuerpo propio constituye un caso claro de esta experiencia de auto-afección: «Siento mi cuerpo que soy yo». Pensamos aquí en las páginas de Edmund Husserl sobre las dos manos que se $\operatorname{tocan}^{14}$, aunque Fabris se refiere más bien a la Fenomenología de la percepción de Maurice MerleauPonty ${ }^{15}$. Sentir no es otra cosa sino «sentirse como otro», aunque en este “otro" se percibe a «sí mismo que se manifiesta como otro» ${ }^{16}$. Estas

12 Ibid., p. 27.

13 Ibid., p. 28.

14 Ed. Husserl, Ideen zueiner reinen Phänomenologie und phänomenologischen Philosophie, II, Phänomenologische UntersuchungenzurKonstitution, § 36 («Konstitution des Leibes als Trägerslokalisierter Empfindungen », HUA, Bd. iv, pp. 144-146.

15 M. Merleau-Ponty, Phénoménologie de la perception, I («Le corps»), cap. 2 («L'expérience du corps et la psychologie classique»).

16 Las citas de este párrafo están tomadas de A. Fabris, «Il coinvolgimento degli affetti», p. 30. 
Naturaleza y Libertad. Revista de estudios interdisciplinares. Número 4, 2014. ISSN: 2254-9668

consideraciones pueden extenderse a los sentimientos, por ejemplo a la alegría. En la alegría, de hecho, «aquello que acontece es el encuentro, en mí, de un aspecto propio que me lleva y me conduce, en un cierto modo, más allá de mí mismo», pero sin sacarme fuera de mí como si yo pudiera convertirme en un "objeto" para mí mismo.

Fabris concluye escribiendo que «el carácter más apropiado de cada sentir, de cada emoción, de cada pathos, es [...] una inmediata adhesión a sí» ${ }^{17}$; aunque se trata siempre de una adherencia vivida en relación a «aquello que es». Podemos distinguir a continuación, sobre esta base y releyendo algunas etapas de la historia del pensamiento, los términos "pasión” y "emoción", precisando a la vez la palabra "afecto".

\section{EL VOCABULARIO SOBRE EL AFECTO EN LA HISTORIA DEL PENSAMIENTO}

A lo largo de la historia del pensamiento filosófico, no faltan formulaciones diversas que jalonan las etapas que condujeron a la formación de nuestro tema. Consideremos, desde esta perspectiva, sólo a tres autores: Aristóteles, Kant y Heidegger.

17 A. Fabris, «Il coinvolgimento degli affetti», p. 32. 
Naturaleza y Libertad. Revista de estudios interdisciplinares. Número 4, 2014. ISSN: 2254-9668

\subsection{LA ANTIGÜEDAD GRIEGA (ARISTÓTELES)}

Con frecuencia, el punto de vista del Estagirita, habitualmente sistemático, se detiene únicamente sobre la formalidad lingüística; y, de esta forma, el autor manifiesta su intención de ordenar el vocabulario común, buscando las definiciones más adecuadas. Este esfuerzo, heredero de la actitud de Sócrates, busca servir a la progresiva constitución de un saber científico, esto es, de un saber que evita la ambigüedad del lenguaje común. El vocabulario que nos interesa ahora se precisa en diferentes lugares de la obra del Estagirita ${ }^{18}$, entre los que consideremos con brevedad algunos pasajes: primero de las Categorias, después de la Metafísica y, al fin de la Ética a Nicómaco.

En las Categorias 8, el término "pathetikos" indica una cualidad afectiva advertida por quien recibe o sufre un influjo externo, pero sin que haya una referencia clara a la realidad que ha sido sentida; sino que el término connota más bien la sensación específica producida pero en cuanto que re-sentida. El afecto, de este modo, traduce la voz "pathetikos" y queda definido como si fuera una cualidad que carece totalmente de aspectos ontológicos, a no ser por el hecho de que provoca un cambio en el sujeto afectado, por ejemplo,

18 La explicación que se propone aquí de las tesis del Estagirita sigue el artículo de S. D'Agostino, «La sfera patemica secondo Aristotele. Dalle qualità affettive alla strategia catartica», en: P. Gilbert [ed.], Passione. Indagini filosofiche tra ontologia e violenza, Assisi, Cittadella [Religione e scienze umane], 2007, pp. 9-40. 
«avergonzándose uno se enrojece, y teniendo miedo, pálido, y así sucesivamente» ${ }^{19}$ - lo cual es sin embargo un verdadero "evento".

En los capítulos 19 a 21 de su Metafísica $\Delta / 5^{20}$, una especie de diccionario de términos útiles en el proyecto de metafísica, Aristóteles precisa el léxico respecto al afecto y a las demás voces que entran dentro de su ámbito de comprensión. Según el capítulo 19, el término "diathesis" significa "disposición”, es decir, un modo de ser, escribe Aristóteles, según el lugar, la potencia o la forma. Este aspecto es importante ya que no se puede ser afectado por algo sin estar dispuesto en un cierto modo a ello; la disposición constituye la condición de posibilidad del afecto, al que, de hecho, determina. Para el capítulo 20, la voz "exis", que posee un gran número de significados, quiere decir principalmente "hábito" (“babitus” en latín) y caracteriza «cierta actividad propia de aquello que posee y de aquello que es poseído, como una acción o un movimiento». En esta descripción, se pone el acento sobre la actividad, la cual se desarrolla obviamente bajo la posibilidad predeterminante de la "disposición”, de la que se hablaba en el capítulo 19. La voz "exis" indica a continuación la posesión del producto de la actividad desarrollada; sintetiza en ello el sujeto activo y el objeto producido; por ejemplo, «entre quien posee un vestido [un traje] y el ser poseído del vestido está, en medio, la acción de poseer». La voz "exis" significa también la "diat- 
hesis" de la que se ha hablado en el capítulo anterior, o el «estar dispuesto a» un bien (salud) o un mal (enfermedad). Y "exis" significa, por último, la «parte de una disposición» que sirve para la disposición entera; la "arete”" o la "exis" de una parte vale para el todo; por ejemplo, si una parte del cuerpo está mal, todo el cuerpo está mal; otro ejemplo, la habilidad del cálculo no se determina en un cálculo particular; aquél que calcula bien en un contexto determinado podría seguramente calcular bien en otros contextos. Al fin, según el capítulo 21, el término "pathos", voz que nos interesa y que Aristóteles sitúa en un conjunto lingüístico, tiene un matiz, por así decir, negativo, al indicar una «cualidad según la que una cosa puede alterarse», o «la puesta en acto de esta alteración», o, más aun, las alteraciones dañosas, los grandes desastres. Si entiendo bien el texto del Estagirita, la voz "exis" indica una actividad positiva de composición, mientras que el término "pathos" hace referencia a una pasividad que puede provocar una descomposición de la interioridad humana. La "disposición" del capítulo 19 se presenta entonces indiferente, neutra, susceptible de ser puesta en acto positivamente ("exis") o de sufrir un influjo negativo ("pathos").

En el capítulo 5 del libro II de la Ética a Nicómaco, Aristóteles ofrece otras precisiones diferentes de las de la Metafísica y distingue, por un lado, "pathos" (afecto o pasión, al que siguen los placeres o dolores, todos sufridos: la Ética no se separa aquí de la Metafísica); por otro lado, "dunamis" (la facultad o capacidad, «aquello por lo que se dice que podemos experimentar ciertas 
pasiones», «aquello por lo cual tenemos la posibilidad de sentir piedad»: en la Metafísica, se correspondería con la "diathesis", la "disposición"); y, en tercer lugar, la "exis" o el "babitus". Hablemos primeramente de la "dunamis". Se puede decir que a cada una de las partes del alma le corresponde una "dunamis" particular; por ejemplo, la facultad de conocer tiene una "dunamis" que no es aquella de la facultad de querer, aunque se dé una conexión obvia de estas facultades en la unidad de la psychê. La "exis" sería, sin embargo, algo como la dunamis activa (o la puesta en acto de la dunamis) en el estado clásico de la pura "disposición” o "capacidad de”, “carente" en ello de perfección. Cada facultad está, de hecho, "dispuesta a" o "para”; es decir, está predispuesta, es “capaz de”. Esta predisposición, según Aristóteles, puede convertirse en una "exis", por ejemplo mediante la regular imposición de una educación que elimina algunas disposiciones impuestas con anterioridad y favorece otras mejores, esperamos... La "energeia" de la facultad pone en acto esta "exis", cuyo estado es así mixto: al mismo tiempo una disposición en espera y una puesta en acto de esta disposición. Aristóteles manifiesta después que la "energeia" puede alterar la facultad, por cuanto, sin destruirla, la hace pasar a un nuevo estado.

Existen, según la Ética a Nicómaco, otros significados de "pathos" que tocan específicamente el sufrimiento y el dolor; el Estagirita no se distancia en 
Naturaleza y Libertad. Revista de estudios interdisciplinares. Número 4, 2014. ISSN: 2254-9668

este punto del discurso de la Metafísica, manifestando así la orientación globalmente negativa de su pensamiento sobre el "pathos" en general. La educación de la "exis" puede sin embargo imponer un sufrimiento subjetivo, con una intención positiva; el afecto de la educación tendrá en este caso una resonancia negativa, aunque, en realidad, acabará siendo positiva.

\subsection{LA MODERNIDAD (KANT)}

De la obra de Kant tomaremos dos textos significativos, como son algunos pasajes de la Crítica del juicio y de la Antropología en sentido pragmático. Hagamos primero, sin embargo, una observación. La orientación kantiana no es obviamente aquella de Aristóteles; ya que el filósofo alemán se sitúa en medio de la problemática moderna, que se preocupa por el horizonte objetivo de las experiencias científicas, morales y estéticas. Según el Kant-Lexicon de Rudolf Eisler, el término "afecto" (“Affektion”), ya en la Crítica de la razón pura, de 1780, denota en general la relación de la cosa en sí con el sujeto que la percibe; lo cual significa que el sujeto afectado no es el «creador de sus sensaciones» $»^{22}$ La categoría de causalidad no sirve pues para pensar esta relación entre la cosa en sí, en cuanto fundamento de nuestras sensaciones, y la subjetividad autónoma; la cosa en sí permanece desconocida para nosotros en cuanto subjetividad en sí (esta tesis no hace sino prolongar aquella que ya aparecía en las Categorías de Aristóteles). El afecto es más bien una "fuerza"

22 R. Eisler, Kant-Lexikon, Paris, Gallimard, 1994, p. 10. 
de A (la cosa en sí) que se impone a B (el sujeto que percibe: el afecto propiamente dicho), que provoca la resonancia de A sobre B de tal manera que B pueda tomar conciencia de la resonancia y de sí mismo. Debemos obviamente distinguir los afectos del cuerpo y de la mente. El tiempo y el espacio, o las formas a priori de la sensibilidad son todos ellos afectos que se imponen a la sensibilidad en cuanto facultad de la mente; la conciencia no accede sin embargo a ellos. Ahora bien, el afecto mental se revela sublime porque, aunque fugaz como el tiempo que pasa (está ligado al cuerpo y no a la razón, vinculado por ello a la naturaleza finita del hombre), permanece, con todo, abierto (o dispuesto) a lo indefinido. E1 afecto es sublime porque es finito, a la vez que abierto indefinidamente. Existe en él una huella de lo excesivo. E1 afecto puede, por este motivo, ser utilizado por la razón que toma de él la energía para su dinamismo.

En las «Observaciones generales» del § 29 de la Crítica del juicio, de 1790, se determina el vocabulario del cual buscamos ahora el significado. Aunque su doctrina no parece traicionar ciertamente las posiciones de la primera Crítica, es decir, el interés por las formas a priori de la subjetividad, las precisa. Kant escribe una larga nota ${ }^{23}$ en la que distingue entre "Affecten" ("afectos") y "Leidenschaften" ("pasiones"). Los "afectos" tienen específicamente una relación con el sentimiento (“Gefühl”) y, según Kant, están así ordenados a una 
subjetividad, sin intenciones o proyectos precisos; pueden ser «impetuosos e involuntarios», pero, de todas formas, «en el afecto, la libertad de ánimo es [solamente...] impedida». Las "pasiones" son, por el contrario, unas «inclinaciones que hacen más difícil o imposible cada posibilidad de determinación del libre arbitrio mediante principios [...]; así, en la pasión, [la libertad] es destruida». En verdad, el afecto no destruye la operación racional y libre, sin embargo impide su justo funcionamiento, hecho que Kant explica de este modo: el afecto es «el movimiento de ánimo que hace incapaz de emprender una libre reflexión sobre los principios con el fin de poder determinarse según estos». El trabajo de la razón sobre los afectos es entonces posible. Kant define a continuación dos tipos de pasiones: la "pasión" ("Leidenschaft") propiamente dicha y la "emoción"; el criterio para distinguir entre ambas es de carácter temporal, pero no sólo, ya que a la vez se refiere a su grado de intensidad. De esta manera, el término "emoción" denota la «sensación en la que la satisfacción es sólo suscitada por una interrupción momentánea y por la fortísima transferencia que le sigue»; la emoción es, por tanto, tempestuosa, irreflexiva; nace por ejemplo de la indignación, o de la ira; dura sólo un instante, y por ello su fuerza puede irse desvaneciendo progresivamente hasta convertirse en un simple afecto y una fuente de "sentimentalismo" que no destruye las posibilidades de la razón. De modo contrario, la pasión es duradera y constituye una auténtica "enfermedad" de la razón, a la que retiene como sierva durante un largo periodo temporal. 
La Antropología en sentido pragmático, de 1798, retoma el tema pocos años después de la Crítica del Juicio. Según el § 74, el término "Affekt" significa la resonancia subjetiva de una sorpresa causada por una impresión intensa, imprevista, que abole la compostura del alma, aunque sólo por un instante; precipitada, se manifiesta carente de sabiduría e imprudente, ahora bien, sin crear grandes peligros. La doctrina es la de la Crítica del juicio. El $§ 79$ («Observación general») hace notar cómo algunos sentimientos que proceden del cuerpo parecen estar emparentados con las emociones, por su duración instantánea, aunque en realidad, contrariamente a las emociones, no dejan huella alguna, por ejemplo el estremecimiento de miedo. Según Kant, podemos suponer que, en virtud de la memoria corporal, exista una continuidad entre la emoción y la pasión. El § 80, confirmando de nuevo la doctrina de la Crítica del Juicio, reconoce que la pasión es un tipo de inclinación. La inclinación, según Kant, se identifica con el deseo de los sentidos; y así, cuando llega a erigirse en una regla indebida de la práctica, destruye la razón. La pasión es una inclinación exagerada, exclusiva, de un tipo distinto al de la emoción y constituye una auténtica enfermedad de la razón, a la que desvía de su correcta dirección. Al fin, en el § 81, Kant concluye distinguiendo con fuerza entre emoción y pasión: «La emoción daña momentáneamente la libertad y la auto-posesión subjetiva; la pasión, en cambio, renuncia a la libertad y encuentra su placer y su satisfacción en el servilismo». 
En resumen, diremos que, en el afecto, la subjetividad accede a la conciencia de sí y de una intencionalidad no definida. En las dos formas de emoción (instantánea) y pasión (duradera), la conciencia se abre en cambio a lo imprevisto, se libera en un cierto modo de sí misma y también, en el caso de la pasión, de la razón. El afecto ofrece así una posible apertura a «aquello que es» distinto de la subjetividad racional, sin que, sin embargo, conduzca de modo seguro a un conocimiento crítico o verdadero: no obstante, su horizonte es ya ontológico. La razón, por lo contrario, está auto-referida, autogestionada, sobre todo cuando se pretende puramente formal, determinada sólo en virtud de unas leyes propias. Al someterse el afecto a la razón, la forma "trascendente" que pertenece a la estructura fundamental del efecto desaparece en el interior de una razón toda ella inmanente, gestionada únicamente por sí misma. Parece, por tanto, que, en la doctrina de Kant, la apertura ontológica de la razón pueda ser reforzada por el afecto emotivo, aunque evidentemente no por la pasión.

\subsection{MARTIN HEIDEGGER}

La propuesta de Heidegger pertenece a una nueva etapa del pensamiento. La cultura de su tiempo no se preocupa por la formación de la objetividad del conocimiento, cuanto del acontecer del sentido en el conocimiento. Para Kant, la ontología se había convertido en un problema, ya que ésta podría estar vacía de significado; en cambio, para Heidegger, la ontología está pingüemente cargada de significado. La intencionalidad, para Kant, está por 
construir mediante un análisis de la estructura de las potencias subjetivas; para Heidegger, está por re-construir a partir del "Sein" que "es gibt".

El tema del afecto es rico en la reflexión de Heidegger, sobre todo durante los años de Sein und Zeit, publicado en 1927, en los que recibe la influencia de Max Scheler. El término "Stimmung", característico de la afectividad, significa "tonalidad afectiva" ("Stimme” significa "voz" y "tono”, una modalidad del sentido; "Stimmung" se traduce habitualmente como “acuerdo", o "armonía” en el sentido de un "acorde musical”). La "Stimmung" caracteriza el "Dasein" arrojado en el mundo, al mundo, de "acuerdo" con el mundo. Otro término, cercano al precedente, es "Befindlichkeit", que significa literalmente "encontrarse" en el mundo; tenemos aquí un matiz de indisponibilidad o de destino, de "facticidad", que pone en evidencia que el "Dasein" no dispone de su "ser arrojado en el mundo". He aquí el significado clásico de "afectividad", la "disposición" de quien está arrojado en el mundo de acuerdo con éste, aunque con un matiz de "facticidad".

La angustia, afecto muy significativo en Sein und Zeit, constituye una aparición privilegiada de la "Stimmung" según el modo propio de la "Befindlichkeit". Sin embargo, revela principalmente la indisponibilidad del ser respecto al mundo: en este sentido, es similar a tantos otros modos de percibir el mundo, de dejarlo aparecer, de ser en el mundo, y ofrece a tales modos, según Heidegger, un aparecer "modélico". La Stimmung no es, por tanto, algo solo interior; antes bien, presenta un "eco" en mí del mundo que me 
aparece o, mejor dicho, un modo peculiar de la presencia "destinal" del yo en el mundo y, por ello, del mundo en mí. La "Stimmung” constituye así una forma de intencionalidad subjetiva que el mundo determina en mí según su propio criterio, pero siempre de acuerdo a las posibilidades de mi "facticidad".

No entraré en la discusión del tema de la "Stimmung" y de la "Befindlichkeit" en la inmensa literatura heideggeriana. Me contentaré con referir ahora un artículo de Marc Richir ${ }^{24}$. Richir percibe que, en Heidegger, no existe reflexión alguna sobre la "pasión" como tal, dado que el filósofo alemán no se preocupa de elaborar una auténtica filosofía del cuerpo animado, de la carne, del "Leib" en el lenguaje de Husserl, que se debería distinguir del "Körper" (“cuerpo" diverso de "carne”); Heidegger dice sólo que el cuerpo animado está en el mundo, ignorando la distinción husserliana y perdiendo de vista, por lo tanto, «la enseñanza misma de Husserl» ${ }^{25}$. Sartre pretende corregir a Heidegger sobre este punto, primero en Esquisse d'une théorie philosophique des émotions, un texto de 1937, y después en L'être et le néant, de 1943. La afectividad originaria sería para Sartre la «conciencia no tética [es decir, no consciente de modo explícito] del cuerpo» ${ }^{26}$. Esta conciencia sin

24 M. Richir, «Affectivité», en: AA.VV., Dictionnaire de la philosophie, Paris, Encyclopaedia Universalis e Albin Michel, 2006, pp. 28-47.

25 Ibid., p. 40.

26 Ibid. 
palabra es vida contingente, por sí, pura facticidad corporal, de acuerdo con la idea del "Körper" husserliano. Según Jean-Paul Sartre, que propone una filosofía determinada por su contexto personalista (que él no estimaba desde luego), el deseo nace de la diferencia vivida entre el cuerpo no tético o para mí (esto es, la facticidad corporal pre-explícita)y el cuerpo que el otro objetiva o propone como tesis (esto es, el cuerpo tético o hecho explícito por el otro o por causa suya). Cuando percibo esta diferencia, mi "afectividad" se convierte en "deseo" y se hace posible así una consideración de la "pasión" en cuanto tal. Maurice Merleau-Ponty, en Le visible et l'invisible, publicado tres años después de su muerte, en 1964, dice que la afectividad originaria se encuentra en el "Leib"; la suya es de hecho una filosofía de la carne, de la encarnación. No obstante, en Sartre como en Merleau-Ponty, según Richir, se mantiene como un "iatus" entre el afecto y la pasión, por cuanto su reflexión no considera el cuerpo distinto de la carne.

Richir lleva a cabo además otra crítica a Heidegger, que confirma aquella otra inicialmente realizada: en la literatura del filósofo de la Selva negra, la "Stimmung", enlazada con el mundo, es también fuente de temporalización, del pasado, del presente y del futuro. ¿De dónde procede entonces el aburrimiento, que es la parálisis del tiempo y de la vida, sin pasado, presente ni futuro, el aburrimiento metafísico? Pareciera que la reflexión sobre el aburrimiento se tenga que poner en relación con algo aun no conocido, es decir, en relación con esta repetición meta-temporal que encontramos inevitable- 
Naturaleza y Libertad. Revista de estudios interdisciplinares. Número 4, 2014. ISSN: 2254-9668

mente en la "anamnèsis" platónica. El aburrimiento no puede por ello constituir un estado del "Dasein” en el mundo ${ }^{27}$. Para Jean-Luc Marion ${ }^{28}$, el aburrimiento es también una "disposición" sin mundo, sin intencionalidad; purifica por ello en la "nada" los aspectos de la angustia que permanecían todavía intencionales en la obra de Heidegger, de quien revela el nihilismo de su propuesta ontológica. Señalemos que Heidegger habla en Sein und Zeit de la angustia, y unos años después del aburrimiento, como si la primera reflexión no fuese ya bastante radical dentro de un proyecto que revela un nihilismo creciente.

\section{AFECTO, EMOCIÓN, PASIÓN Y SENTIMIENTO}

Una vez trazado este recorrido histórico, propongo a continuación una reflexión sistemática, en la que serán precisadas dos categorías: por un lado, la categoría de afecto (en el que se distinguen emoción y pasión) y, por otro lado, la de sentimiento.

27 Heidegger ha escrito ampliamente sobre el aburrimiento; véase, por ejemplo, Die Grundbegriffe der Metaphysik. Welt, Endlichkeit, Einsamkeit, § 37-38 (GA 29), un curso de invierno en 1929-1930, poco después de la edición de Sein und Zeit.

28 J.-L. Marion, Réduction et donation. Recherches sur Husserl, Heidegger et la phénoménologie, Paris, Presses Universitaires de France, 1989, pp. 280-289. 
Naturaleza y Libertad. Revista de estudios interdisciplinares. Número 4, 2014. ISSN: 2254-9668

\subsection{AFECTO}

$\mathrm{El}$ afecto, con un matiz propio de finalidad y de energía, posee una dimensión temporal que, sin unas ciertas distinciones internas, no podría darse.

La fuerza impositiva de la emoción invade en un instante todo el campo de la conciencia, la cual es aspirada en una especie de vórtice interior, en el que la subjetividad se siente abandonada a las pocas fuerzas que le quedan. Por el contrario, la pasión tiene una larga duración; nace habitualmente de una emoción o de una sucesión de emociones similares, superando progresivamente el sentimiento de abandono en la soledad y retomando así la fuerza y el contacto con la realidad, también con aquella que previamente ha provocado emoción. Notemos asimismo que no se da la pasión sin implicar o utilizar a la razón, o al menos a algunos de sus aspectos, en primer lugar, a los que presiden la organización del tiempo, la forma de coherencia de la que se pretende revestir la propia existencia. De esta manera, frente a la emoción que parece totalmente irracional, la pasión no es desorganizada. Sobre este punto, prefiero no seguir a Kant.

\subsubsection{LA EMOCIÓN}

El afecto puede ser definido de este modo: «puesta en práctica de la adaptación del modo de ser del sujeto a la realidad». Cuando el afecto posee un aspecto de inmediatez, de instantaneidad, es decir, cuando es una emoción, se puede definir así: «imposición en el instante de un cambio de dirección». Se habla ahora de una emoción "choc", que distinguiremos más 
tarde de la emoción "sentimiento". Propongo profundizar el significado de esta emoción "choc". Experimentamos con frecuencia la imposición en el instante de la maravilla o del estupor —una actitud que los filósofos han señalado habitualmente en el comienzo mismo de cada empresa o investigación científica. Podemos conectar además la emoción y el nacimiento de la conciencia, ya que la emoción provoca, de hecho, una toma de posesión de sí, o al menos, reenvía a la conciencia (herida o entusiasta) de sí. El "choc" de la emoción pone a la persona emocionada delante de sí, es decir, despierta la conciencia a ser ella misma y también al deber de clarificarse, al deber de tomar posición, de decidir, no tanto sobre su orientación, cuanto de decidir simplemente orientarse.

A la toma de conciencia provocada por la emoción "choc" le sigue un cambio orgánico provocado por el evento sufrido y chocante. Cuando, por ejemplo, veo un accidente y mis piernas comienzan a temblar, tomo conciencia de mi miedo y después del peligro que yo mismo habría podido sufrir. Una interpretación de la emoción, que supone la existencia de un lazo de unión entre la causa de la emoción y el comportamiento fisiológico, se realiza en un ambiente de estrecha racionalidad mecánica. Así, en Lo voluntario y lo involuntario, Ricœur parece comprender la esencia de la emoción "choc" a través del modelo del mecanicismo comportamental. El filósofo francés percibe, de hecho, que este tipo de emoción constituye una novedad provocada por una ruptura por la cual el espíritu libre y maestro de sí desaparece, aplas- 
tado bajo el impulso violento del cuerpo. Escribe Ricœur: «La emoción"choc" constituye un auténtico trauma del querer: la función de la emoción queda completamente borrada; el desorden conquista en un cierto sentido su independencia y al mismo tiempo, toda su in-inteligibilidad. El hombre se convierte en alguien irreconocible: es grito, temblor, convulsión [...]. A partir de este momento, el cuerpo emprende su camino por sí mismo y el caballero es arrojado; de esta forma, puede parecer que la emoción no sea más que un epifenómeno del cuerpo» ${ }^{29}$ que aniquila todo querer libre o autónomo. Ricœur concluye: «me parece que la idea de espontaneidad de la conciencia, ha de ser sustituida por la idea de una pasión del alma, que es obra del cuerpo [...] La emoción provoca así la inherencia vital del cuerpo en la conciencia, mientras la insurrección del cuerpo afecta inmediatamente a los pensamientos y esboza una acción agitada que demanda el querer» ${ }^{30}$.

29 P. Ricœur, Le volontaire et linvolontaire, Paris, Aubier-Montaigne, 1950, pp. 252 y 254.

30 Ibid., p. 259. Podemos citar otros textos de Ricœur: «La función más rudimentaria de la emoción es la sorpresa o el rapto (la admiración cartesiana); en un segundo momento, ésta se complica a causa de las formas emotivas de la imaginación afectiva, por medio de las cuales anticipamos algún bien o algún mal. Ésta alcanza su punto culminante en la alerta del deseo y encuentra su coronación en la moción de la alegría o de la tristeza que sancionan la posesión de algún bien o de algún mal» (238). Ricœur se refiere a la sorpresa de acuerdo con su primera definición de este manera: la sorpresa es un «fenómeno circular entre el pensamiento y el cuerpo» (238) que "desorganiza el curso regular y adecuado del pensamiento y de la vida» (239). El segundo momento de la sorpresa suscita otra consideración definitoria: «La emoción se nutre de la resonancia corpórea; el robo del conocimiento se halla en el camino de reflexión 
La emoción que nace con lo inesperado, con la novedad, la sorpresa, no es sin embargo solamente pasiva, una vivencia provocada por un "choc" destructor de la libertad. Puede generar una acción, una adaptación progresiva a las situaciones, y sobre todo la transformación de éstas. En la emoción, el cuerpo sufre en el alma, en la conciencia de sí, y abre un posible porvenir para la libertad y su compromiso en la realidad. Darwin la define como «manifestación de la lucha por la adaptación», una adaptación que debemos pensar de modo recíproco: no sólo desde el sujeto, sino también desde la realidad objetiva. Entramos ahora en una dimensión de duración temporal que no es ya propia de la emoción y que remite a la pasión.

\subsubsection{LA PASIÓN ${ }^{31}$}

Por pasión entendemos, entre otras cosas, un modo de gestionar las relaciones con nuestros interlocutores, una especie de aparición que expresa nuestro ser y nuestra auto-conciencia en relación con ellos. La lógica formal

del estupor corpóreo sobre el pensamiento» (239). Por ello, diremos que «el cuerpo amplifica y exalta el instante del pensamiento» (239). Debemos reconocer por tanto que existe una doble anticipación afectiva del pensamiento, del conocer: desde el punto de vista de la toma de conciencia, la emoción anima, en primer lugar, el conocimiento directo y, en segundo lugar, la emoción surge ella mima en un cuerpo preparado; desde el punto de vista de la generación de los fenómenos, la secuencia es contraria: la emoción da «resonancia a una amplificación corpórea al juicio de valor rápido y no querido» (241).

31 El texto sobre la "pasión" retoma y adapta una parte de mi artículo «Ragione, realtà, compassione», en: P. Gilbert (ed.), La passione, pp. 155-179 (167, 170-171). 
clásica constituye, sin embargo, un modo de hablar que no tiene en cuenta a los interlocutores; pretendidamente apática, esto es: $\sin$ pathos; se espera que funcione por sí misma, como una calculadora. La investigación formal, no obstante, ¿estará ayuna de cualquier modo de pasión? La tradición racionalista así lo haría pensar, más aun así lo proclama en todas sus expresiones. Sin embargo, yo no he encontrado jamás a un lógico que no se "interese" pasionalmente por la lógica.

Mi hipótesis pretende mostrar cómo la pasión sería la experiencia originaria de la conciencia. Para sostener tal hipótesis, debo precisar aquello que entiendo por pasión. La pasión es obviamente operativa, activa ya que responde a una solicitación o a una provocación. Desde el punto de vista de esta estructura, pasión y emoción se parecen, pero esta última actúa sobre el psiquismo del sujeto hasta bloquearlo, mientras que la pasión empuja el organismo a la acción, a emitir una respuesta frente a aquello que lo ha tocado. Está claro que tal respuesta se corresponderá con la potencia de la emoción. Quizá tendríamos que reconocer aquí una cierta inversión del vocabulario común: de hecho, el término "emoción” parece significar un movimiento de sí mismo que ha sido convocado fuera de sí mismo, de sí mismo que sufre un movimiento ("moción”) que lo lleva fuera de sí; el término "pasión" evoca sin embargo una pura pasividad; la persona apasionada parece en efecto haber perdido la posesión de sí. El uso lingüístico ha invertido extrañamente estos matices, lo cual significa una permanente 
superposición del significado de un término en el otro. Mi análisis pone en evidencia también que a estos términos se añaden algunos aspectos temporales, que los mismos no expresan directamente. Si la emoción surge en el instante de su provocación, en presencia de aquello que emociona; la pasión, en cambio, actúa de modo más duradero, también cuando no está presente su meta; más aun, prescinde habitualmente de la presencia de lo emocionante. La emoción, poniéndose sustancialmente en presencia aquello que es, posee un aspecto físico y accidental que la pasión no conoce. La pasión es más libre, y entonces humana.

Debemos precisar ahora el tipo de pasión que, más que las demás, es capaz de confrontarse con la realidad. Son tantas las pasiones, como muestran las meditaciones de los filósofos y las investigaciones de los psicólogos. Descartes atribuye a la admiración el primado de las pasiones; según Spinoza sin embargo, será la alegría la primera entre todas. Para el cristiano, es el amor intencional y relacional, atento y fiel a la relación en la duración histórica. En la tradición filosófica, a la realidad "objetiva" le corresponde una intención "subjetiva”, espiritual, que la Edad Media explicaba como un deseo, en clave, por tanto, de afectividad. Ahora bien, aún el conocimiento tiene que ser considerado bajo el aspecto de un dinamismo que no termina nunca, y por eso de la insatisfacción, o también de la felicidad sin sus conquistas provisionales. 
Naturaleza y Libertad. Revista de estudios interdisciplinares. Número 4, 2014. ISSN: 2254-9668

\subsection{SENTIMIENTO}

Pasemos ahora a la consideración de la categoría de "sentimiento". Existen muchas definiciones que ponen de manifiesto una relación entre el sentimiento y el conocimiento, sobre todo el conocimiento de sí mismo. Según la «Cuarta consideración» de las Meditaciones para disponerse a la bumildad y a la penitencia de Nicolás Malebranche, «aunque mi alma está muy por encima de mi cuerpo, siento, sin embargo, que el placer, el dolor y todos los demás sentimientos que poseo, se llevan a cabo en mí independientemente de mí mismo, más aun, algunas veces se llevan a cabo a pesar de todos los esfuerzos que opongo. De este modo, no puedo dudar que exista algo diverso del alma, que vivifica el sentimiento del alma» ${ }^{32}$. En Acerca del hombre considerado como bijo de un padre pecador, el mismo autor escribe que «el sentimiento confunde el conocimiento, ya que modifica el espíritu y lo hace participar de su capacidad que es finita ${ }^{33}$. Todas estas consideraciones o definiciones conciben el sentimiento conforme al modelo de la emoción que hemos descrito, elevándolo a un nivel cognitivo. Al fin, en su obra Búsqueda de la verdad, Malebranche afirma que el alma se conoce mediante un «sentimiento interior ${ }^{34}$ que él interpreta en contraste con el modo cartesiano de la

32 N. Malebranche, Euvres, t. XVII-I, Pièces jointes. Écrits divers, Paris, Vrin, 1960, «Méditations pour se disposer à l'humilité et à la pénitence», p. 394.

33 Ibid., «De l'homme considéré comme fils d'un père pécheur», p. 398.

34 Ibid., t. I, Recherche de la vérité, liv. I-III, ${ }^{2} 1972$, p. 415. 
Naturaleza y Libertad. Revista de estudios interdisciplinares. Número 4, 2014. ISSN: 2254-9668

idea clara y distinta, evidente del cogito. El sentimiento constituye, pues, una percepción confusa de sí mismo y de las cosas, que es el único conocimiento posible en ciertas circunstancias.

En un artículo dedicado al «Sentimiento», Olivier Reboul define así el sentimiento: «es ante todo el acto y el resultado del sentir, el cual indica la toma de conciencia inmediata, sin intermediario y sin distancia, de las cosas y de nosotros mismos: el objeto del sentimiento es siempre aquello que nos “toca” " ${ }^{35}$.Este sentimiento tendrá un cierto matiz de creencia, el cual se expresa, por ejemplo cuando digo que «no siento que las cosas sean así», quedando de manifiesto que carece de razones claras y distintas. Para Blaise Pascal, «el corazón posee sus razones, que la razón no conoce», el sentimiento está referido estrechamente al "corazón” y no a las razones de la razón determinante y predicativa. Los sentimientos, con frecuencia, invitan entonces a la acción, verbi gratia el sentimiento de amor. No se someten sin embargo a una razón que esboza unos planes bien organizados de acción y no dan lugar, por ello, a un conocimiento científico argumentado y progresivo. El sentimiento constituye, sin embargo, un modo específico de estar en el mundo y de orientarse dentro de él, un modo duradero, que incluye a todo el hombre y a todo el espacio de su conocimiento.

35 O. Reboul, «Sentiment», en Aa.Vv., Dictionnaire de la philosophie, pp. 1856-1862 (1857). 
Según Reboul, el sentimiento en general es un modo de conciencia intencional (más que de conocimiento tético); constituye la conciencia de una presencia irreductible a la razón y al saber determinado por la causalidad. El entendimiento, o la razón stricto sensu, capta las esencias formales; el sentimiento revela en cambio la existencia. Malebranche objetaba de modo parecido contra Descartes: el sum no está ligado al cogito como si fuera su representación racional. La presencia sentida tiene de por sí un valor, una significación que ninguna determinación racional podrá fijar ya que pertenece a nuestra experiencia de vida de los sentimientos en relación con el valor de eso que toca, física o mentalmente. De este modo, al sentimiento, le sigue siempre "algo", de forma análoga a la de la conciencia que, según Husserl, conoce siempre "algo" (aunque no se pueda determinar el valor científico de la verdad de tal conocimiento). El sentimiento contrario, esto es, estar en el vacío, confirma esta tesis: para el sentimiento desesperado del vacío, sentimiento éste que se conoce como "desidia", no existe nada que tenga una verdadera importancia; es esta "nada" lo que asusta, como si fuera "algo". Así, el sentimiento implica siempre la conciencia de un compromiso, de una participación, positiva o no, no importa.

\section{CONCLUSIÓN}

La tradición filosófica ha reconocido siempre que el conocimiento es intencional, que, al dirigirse a una cierta "presencia", tiende hacia "algo" que es 
específicamente una realidad sensible o mental. De esta manera, cada conocimiento se pone en marcha con la acogida de una "presencia" sensible o mental, es decir, no sólo por la recepción de una experiencia sensible y su explicación mecánica. La idea de "presencia" es amplia. Sin embargo, el vocabulario que expresa las dinámicas cognoscitivas remite con frecuencia a la experiencia de lo sensible, también cuando se trata de realidades inteligibles. Por ejemplo, el término "saber" procede de "sabor", "contemplar" de "mirar atentamente", o la misma voz "idea" viene del griego "eidein", que se traduce por "ver".

Por ello, el intelecto no entiende la propia esencia si no asume una cierta referencia a la sensibilidad. La filosofía contemporánea, en la fenomenología de Edmund Husserl y sobre todo a partir de los textos publicados bajo el título de Ideas II: Investigaciones fenomenológicas sobre la constitución ${ }^{36}$, ha vuelto sobre el tema de la sensibilidad, analizando todos los aspectos con un rigor y una profundidad que los estudios de fisiología ignoran. Entre estos aspectos, se sitúa el espacio propio de la intersubjetividad. La Fenomenología de la percepción de Merleau-Ponty ${ }^{37}$ se erige, en este sentido, en un testimonio impresionante. $\mathrm{Al}$ respecto, el examen de la experiencia sensible se enriquece 
con la atención a la intersubjetividad, concretamente a los aspectos de la empatía $^{38}$, y, a continuación, de la misma afectividad.

Otros términos que difieren de los ya señalados ("saber", "contemplar" o “idear") expresan, por su parte, nuevos matices significativos que precisan la comprensión de nuestra actividad cognoscitiva, pero sin referirse a la experiencia sensible, por ejemplo: "meditar", "reflexionar", "pensar". Estas actividades, solitarias, que no pueden desenvolverse en la precipitación y la prevención ${ }^{39}$, exigen por nuestra parte una fuerte capacidad de crítica de sí y, al mismo tiempo, de atención a eso que aparece, a su presencia especifica. La meditación, que no logra satisfacer cuando saltamos de un tema a otro, requiere, antes bien, una disponibilidad mental, o espiritual, que asegure una cierta permanencia en el dominio de lo meditado, una disponibilidad para permanecer. Con la reflexión ocurre algo similar, aunque bajo un aspecto más característicamente racional, ya que ésta no ignora las condiciones y los beneficios de la argumentación concluyente, es decir, de nuevo, del permanecer en la presencia delo reflexionado. Por último, la actividad del pensamiento, que se extiende durante un tiempo que no se puede delimitar a priori, es, en un cierto sentido, más libre que la reflexión y, por consiguiente, más creativa; pero tiene que elaborar sus juicios con prudencia, integrando con atención los diversos aspectos de lo pensado.

38 Cfr. Edith Stein.

39 R. Descartes, Discours de la méthode, éd. Adam et Tannery, Paris, Vrin, 1996, p. 18. 
Se debe insistir sobre el hecho de que la afectividad no es sólo mera subjetividad: será obviamente una "capacidad", una "potencia" de la subjetividad, que, no obstante, no viviría si no se pusiese en relación con una realidad sensible o mental. Los términos que despliegan los trazos de nuestra afectividad cognoscitiva evocan los mundos de lo sensible y, al mismo tiempo, de lo mental. No podemos separar estas dos referencias. El trabajo específicamente intelectual, argumentativo, no puede dejar de lado la experiencia sensible, que atrae hacia una realidad que no es sensible, cuanto mental, o más aun, espiritual. No hay "pensar" sin "sabor". La emoción "choc", según hemos dicho ya, está en el origen mismo del conocimiento, provocado por la maravilla, por el estupor del espíritu a causa de la ruptura de su mundo mental cotidiano. La emoción debe convertirse a continuación en pasión para llegar a ser fecunda. El coraje de conocer, es decir, de estudiar, constituye un cierto modo de pasión lenta, testaruda, fiel y, con frecuencia, sometida a la ilusión, y por eso llamada a una verificación prudente e inteligente. Sin emoción y pasión, dos aspectos esenciales del afecto, no existe conocimiento alguno.

El conocimiento está aún penetrado de un sentimiento. Muchos estudiosos no estarán de acuerdo, obviamente, con esta tesis ya que el término "sentimiento" ha recibido en la cultura contemporánea ciertos significados subjetivistas y románticos que lo alejan de su sentido más profundo, es decir "sentirse en casa", "habitar en la verdad", o vivir en el "filein" de los Griegos. El sentimiento no se da sin una disposición a la acogida de aquello que es, 
Naturaleza y Libertad. Revista de estudios interdisciplinares. Número 4, 2014. ISSN: 2254-9668

aunque no se pueda determinar "objetivamente". De modo similar, según la tesis ya presentada de Malebranche, el cogito cartesiano expresa un sentimiento de sí, y no una idea clara y distinta. Ahora bien, existe un ligamen entre sentimiento y deseo. Ambos son dinámicos y se orientan hacia una realidad que, de cualquier forma, está más presente en el sentimiento que en el deseo; como si el sentimiento pudiera sostener la fidelidad del deseo; y, como si el deseo, en vistas a una posible determinación de lo "objetivo", ayudase recíprocamente al sentimiento a permanecer en la rectitud.

Paul Gilbert, S J

gilbert@unigre.it. 